\title{
Estado de hipercoagulabilidad y complicaciones trom- boembólicas en el paciente con infección por SARS-CoV-2
}

\section{Hypercoagulability status and thromboembolic complications in pa- tients with SARS-CoV-2 infection}

Recibido: $18 / 06 / 2021$

Aceptado: 22/07/2021

\author{
Nurian Alejandra Hernández Loyo \\ Universidad San Carlos de Guatemala \\ nuriana.hernandez20@gmail.com \\ https://orcid.org/0000-0001-6774-4272
}

\section{Referencia del artículo}

Hernández Loyo, N. A. (2021). Estado de hipercoagulabilidad y complicaciones tromboembólicas en el paciente con infección por SARS-CoV-2. Revista Diversidad Científica, 1(1), 15-24.

DOI: https://doi.org/10.36314/diversidad.v1i1.3

\section{Resumen}

OBJETIVO: describir el estado de hipercoagulabilidad y complicaciones tromboembólicas que se presentan en el paciente con infección por SARS-CoV-2. MÉTODO: se recopiló información de Pubmed, Asociación Americana del Corazón, Sociedad Internacional de Trombosis y Hemostasia, libros y revistas médicas mundiales donde se encontraron metaanálisis, artículos y guías sobre el estado de hipercoagulabilidad y complicaciones tromboembólicas que han sido documentadas en el paciente con infección por SARS-CoV-2. RESULTADOS: la coagulopatía asociada a COVID-19 es consecuencia de la tormenta de citocinas desencadenada por la respuesta inmune ante el SARS-CoV-2, la cual provoca lesión endotelial e hiperactivación de la cascada de la coagulación que culmina con generación de fibrina a nivel vascular, favoreciendo así, la estasis sanguínea. CONCLUSIÓN: las principales complicaciones tromboembólicas en el paciente con SARS-CoV-2 han sido la trombosis venosa profunda, tromboembolia pulmonar, síndrome coronario agudo, evento cerebrovascular isquémico agudo, coagulopatía intravascular diseminada, trombosis aórtica abdominal y torácica, siendo el $80 \%$ tromboembolia pulmonar y $31 \%$ trombosis venosa profunda las más frecuentes y aumentando la mortalidad global hasta en $7 \%$ debido al estado hipercoagulable.

Palabras clave: hipercoagulabilidad, tromboembolia, SARS-CoV-2 


\begin{abstract}
TARGET: describe the hypercoagulable state and thromboembolic complications that occur in patients with SARS-CoV-2 infection. METHOD: information was collected from Pubmed, American Heart Association, International Society of Thrombosis and Haemostasis, books and world medical journals where meta-analyzes, articles and guides about the hipercoagulable state and thromboembolic complications that have been documented in the patient with SARS-CoV-2 infection. RESULTS: coagulopathy associated with COVID-19 is a consequence of the cytokine storm triggered by the immune response to SARS-CoV-2, which causes endothelial injury and hyperactivation of the coagulation cascade that culminates in fibrin generation at the vascular level, thus promoting blood stasis. CONCLUSION: the main thromboembolic complications in patients with SARS-CoV-2 have been pulmonary thromboembolism, deep vein thrombosis, acute coronary syndrome, acute ischemic cerebrovascular event, disseminated intravascular coagulopathy, abdominal and thoracic aortic thrombosis, with $80 \%$ being pulmonary thromboembolism and $31 \%$ deep vein thrombosis the most frequent and increasing global mortality by up to $7 \%$ due to the hypercoagulable state.
\end{abstract}

Keywords: hypercoagulability, thromboembolism, SARS-CoV-2 


\section{Introducción}

La pandemia de la enfermedad COVID 19, originada por el virus del Síndrome Respiratorio Agudo Severo 2 (SARS-CoV-2) ha obligado a los sistemas de salud a estudiar en tiempo real los mecanismos fisiopatológicos de la infección a fin de entender las complicaciones que se pueden presentar en los pacientes y que no se limitan al desarrollo de un cuadro de neumonía. EI SARS-CoV-2 a través de sus proteínas estructurales, utiliza como vía de entrada su unión a los receptores de la enzima convertidora de angiotensina 2 (ECA 2) expresados principalmente pero no de forma exclusiva a nivel pulmonar, ya que también se ha documentado su existencia en otros tejidos como riñón, intestino, corazón, útero, cerebro, glándulas suprarrenales y principalmente en el endotelio vascular, lo que explica la inflamación sistémica con una gran liberación de citocinas que provocan el deterioro rápido, insuficiencia respiratoria, circulatoria y alteraciones de la coagulación las cuales pueden aumentar la letalidad del paciente con COVID-19 hasta en un 7\%. (Vargas-Lara, Schreiber-Vellnagel, Ochoa-Hein, \& López-Ávila, 2020) La presencia de coagulopatía asociada a COVID-19 se caracteriza por daño endotelial local y sistémico, lo cual predispone al paciente a presentar hiperactivación plaquetaria, trombosis e insuficiencia del proceso de fibrinólisis con la subsecuente activación de marcadores inflamatorios como monocitos y macrófagos que generan la tormenta de citocinas que se ha demostrado en el paciente COVID-19 y la cual supone ser la perpetuadora de la promoción del estado de hipercoagulación así como de la producción de un círculo vicioso entre inflamación y trombosis (Gonzáles-Villalva, y otros, 2020).

Es importante entender que el estado de hipercoagulabilidad inducido por SARS-CoV-2 se considera responsable de un aumento de la mortalidad debido a la presencia de eventos trombóticos y embólicos como tromboembolia pulmonar, síndrome coronario agudo, trombosis venosa profunda, isquemia mesentérica aguda, trombosis abdominal aguda o el desarrollo de coagulopatía intravascular diseminada que es precursora de la falla multiorgánica. Es por ello que en respuesta a la crisis sanitaria mundial derivada de la pandemia de COVID-19, es importante reconocer la existencia del estado hipercoagulable y entender los mecanismos fisiopatológicos que llevan a su establecimiento a fin de lograr su detección temprana y monitoreo continuo para diseñar las mejores estrategias terapéuticas y lograr la disminución de la tasa de mortalidad debido a eventos relacionados a la coagulopatía asociada a COVID-19.

La objetividad del estudio fueron describir la patogenia de la infección por SARS-CoV-2, describir el estado de hipercoagulabilidad que se produce en el paciente con infección por SARS-CoV-2, identificar los métodos diagnósticos utilizados en la detección del estado de hipercoagulabilidad en la infección por SARS-CoV-2 e 
identificar las principales complicaciones tromboembólicas que se presentan en el paciente con infección por SARS-CoV-2. Se identificó que el SARS-CoV-2 desencadena la enfermedad de COVID-19 en el ser humano debido a que sus proteínas estructurales $\mathrm{S}, \mathrm{M}, \mathrm{N}$ y $\mathrm{E}$ se unen a los receptores de la enzima convertidora de angiotensina 2 ampliamente distribuidos en múltiples tejidos y a través la liberación de citocinas proinflamatorias provocan la hiperactivación de los eventos de la cascada de la coagulación que llevan a la estasis sanguínea y desarrollo de estado hipercoagulable, el cuál debe ser diagnosticado y monitorizado a través del estudio de los productos de degradación de la fibrina, siendo el dímero $D$, el biomarcador que diferentes metaanálisis citan como el mejor predictor de gravedad y mortalidad de la enfermedad. Además, diversas entidades médicas como la Sociedad Americana del Corazón, Sociedad Internacional de Trombosis y Hemostasia, Sociedad Chilena de Cuidado Crítico han realizado diversos meta análisis y estudios que concuerdan que las principales complicaciones tromboembólicas documentadas en el paciente COVID-19 han sido la tromboembolia pulmonar ( $80 \%$ ) y la trombosis venosa profunda (31\%).

\section{Contenido}

El virus del síndrome respiratorio agudo severo 2 (SARS-CoV-2) pertenece taxonómicamente a la familia Coronaviridae, al igual que otros coronavirus, el SARS-CoV-2 tiene un origen zoonótico. A diferencia del SARS-CoV causante de la epidemia de síndrome respiratorio agudo grave de los años 2002-2004 que tenía como hospedero intermediario a la civeta; con el nuevo coronavirus responsable de la enfermedad COVID-19, aún no se tiene claro cuál pudo haber sido el intermediario, aunque algunos expertos sitúan al murciélago como el directo responsable de transmitir el virus al ser humano (Díaz-Castrillón \& Toro-Montoya, 2020).

Debido a sus proteínas estructurales: proteína espícula (S), proteína de membrana $(\mathrm{M})$, proteína de la nucleocápside $(\mathrm{N})$ y proteína de envoltura (E), el SARS-CoV-2, logra su unión a los receptores de la enzima convertidora de angiotensina 2 de amplia distribución en el ser humano, desencadenando un espectro de enfermedades principalmente respiratorias agudas y crónicas, entéricas, hematológicas y del sistema nervioso central, lo que refleja una capacidad de afectación multisistémica y multiorgánica (Alvarado Amador, Bandera Anzaldo, Carreto Binagui, Pavón Romero, \& Alejandre García, 2020).

La unión del SARS-CoV-2 a los receptores de la ECA 2, se induce en la célula infectada la producción de citocinas proinflamatorias como el factor de necrosis tumoral alfa (TNFa), la interleucina 6 (IL-6) y también, la producción de interferones 
inflamatorios ( $\alpha$ y $ß$ ). Estas citocinas proinflamatorias inducen señales que activan el endotelio atrayendo a células del sistema inmune adaptativo y activando el sistema de complemento que ayuda a opsonizar las células infectadas por el virus y permite una mejor citotoxicidad. La activación masiva del complemento ha sido propuesta como la causa de activación de la trombina y los fenómenos trombóticos que se han observado en pacientes con infección por SARS-CoV-2. (Espinosa-Rosales, 2020) Se ha descrito que los pacientes infectados por SARS-CoV-2 tienden a presentar una respuesta inflamatoria exagerada que se caracteriza por una elevación sostenida de interleucinas $(1 ß, 2,7,8,9,10,17)$ y factor de necrosis tumoral a e interferón $\gamma$ y una disminución en el recuento de células T CD4+ y T CD8+. El cuadro clínico de estos pacientes está acompañado de edema e insuficiencia pulmonar, hepatoesplenomegalia, adenopatías, citopenias, alteración de la función hepática y activación de la cascada de la coagulación (Triana-Bedoya \& León-Cardona, 2020).

Consecuencia de la hiperactivación de la cascada de la coagulación se desarrolla el estado hipercoagulable asociado a COVID-19 o respuesta inmune trombótica asociada a COVID-19 (RITAC), a la cual Gauna y Bernava, le han establecido como criterios diagnósticos la presentación de uno o más de los siguientes criterios: dímero $\mathrm{D}>1000 \mathrm{ng} / \mathrm{ml}$, ferritina $>500 \mathrm{ng} / \mathrm{ml}$, disnea de rápida progresión, hipoxemia refractaria, fenómenos trombóticos y/o shock. (Barco-Moreno, Cuellar-Canizales, Vilas-Cruz, \& Bravo-Rojas, 2020).

Desde el inicio de la pandemia de COVID-19, se han reportado serias complicaciones trombóticas en los pacientes infectados, especialmente en aquellos críticamente enfermos. Las autopsias de pulmón de pacientes que fallecieron por COVID-19 revelan edema alveolar difuso, trombosis y formación de membrana hialina propias del síndrome respiratorio agudo severo. En la infección por SARS-CoV-2 ocurre microcoagulación secundaria a trombosis de la microvasculatura pulmonar. (Mondal, Quintili, Karamchandani, \& Bose, 2020).

Distintos metaanálisis realizados por expertos en el área de hematología como la Sociedad Internacional de Hematología y Trombosis, confirman que la enfermedad de COVID-19 se asocia con un aumento de la inflamación, lo que a su vez aumenta el riesgo de trombosis arterial y venosa. Estas complicaciones pueden aumentar el riesgo de morbilidad y mortalidad e incluyen principalmente: isquemia aguda de las extremidades, trombosis aórtica abdominal y torácica, isquemia mesentérica, síndrome coronario agudo, tromboembolismo venoso, evento cerebrovascular agudo y coagulopatía intravascular diseminada que puede conducir a la falla multiorgánica. El conocimiento de estas afecciones en COVID-19 puede mejorar el reconocimiento y el manejo de estos trastornos trombóticos por parte de los médicos de emergencia (Avila, Long, Holladay, \& Gottlieb, 2021). 
Respecto al diagnóstico del tan temido estado de hipercoagulabilidad asociado a COVID-19, la Asociación Colombiana de Medicina Crítica y Cuidado Intensivo, el sistema hemostático del paciente con infección por SARS-CoV-2, se desplaza de forma notable hacia el lado procoagulante. El patrón más común de coagulopatía observado en pacientes hospitalizados en COVID-19 se caracteriza por elevaciones en los niveles de fibrinógeno y dímero $D$, lo cual está correlacionado con un aumento de biomarcadores de inflamación, como la proteína $C$ reactiva (PCR) y la ferritina. (Sáenz Morales, Rubio, Yomayusa, Gamba, \& Garay Fernández, 2020) De acuerdo con Cochrane Iberoamérica, el dímero $D$ es el biomarcador de elección en el diagnóstico y monitoreo del estado hipercoagulable del paciente con infección por SARS-CoV-2. Los pacientes COVID-19 que tienen peor pronóstico han mostrado unos niveles sanguíneos de dímero $D$ mucho más elevado que los pacientes con enfermedad menos grave. (Cochrane, 2021) Individuos con dímero D mayor de $2000 \mathrm{ng} / \mathrm{ml}$ tienen los riesgos más altos de enfermedad crítica (66\%), eventos trombóticos (38\%), injuria renal aguda (58\%) y mortalidad (47\%). (Rosa, 2020) Expertos de la Sociedad Internacional no consideran que el tiempo parcial de tromboplastina activado (TPTa), se realice como una prueba en todos los pacientes hospitalizados por COVID-19 ya que el 91\% de los pacientes con COVID-19 y TPTa son positivos para anticoagulante lúpico y pueden presentar deficiencia de factor XII de la coagulación (Windyga, 2020).

Respecto al conteo de plaquetas, alrededor del $5 \%$ de los pacientes presentan un recuento de plaquetas menor de 100,000 plaquetas/litro. Sin embargo, también se ha documentado trombocitopenia leve en el paciente con enfermedad crítica, por lo cual no se recomienda al recuento plaquetario como marcador sensible ni específico en el diagnóstico y monitoreo del estado hipercoagulable. Otro biomarcador que ha demostrado efectividad en el monitoreo del estado hipercoagulable ha sido el nivel de fibrinógeno, el cual por niveles inferiores a 1 gramo/litro se asoció a alta mortalidad en pacientes con enfermedad grave en China. (Sáenz Morales, Rubio, Yomayusa, Gamba, \& Garay Fernández, 2020) Como se menciona anteriormente, las complicaciones tromboembólicas derivadas del estado de hipercoagulabilidad que se han encontrado en pacientes con infección por SARS-CoV-2 y que en su mayoría se han detectado en estudios post mortem, han sido tromboembolia pulmonar que se ha presentado hasta en un $80 \%$ de los pacientes, seguido de la trombosis venosa profunda que se presenta hasta en $31 \%$ de los pacientes con formas graves de la enfermedad COVID-19. Según un metaanálisis publicado por la editorial británica BioMed Center, que incluyó 19 artículos realizados por instituciones europeas, se concluyó que los pacientes con COVID-19 pueden tener una mortalidad de $45 \%$ secundaria al desarrollo de tromboembolia pulmonar (Liao, Shao, Chen, \& Hung, 2020). 


\section{Conclusión}

El virus del síndrome respiratorio agudo severo tipo 2 (SARS-CoV-2) causante de la enfermedad COVID-19, tiene proteínas estructurales $S, M, N$ y E, a través de las cuales se une y penetra al epitelio respiratorio a través de la unión con el receptor de la enzima convertidora de angiotensina 2 (ECA 2), produciendo citocinas proinflamatorias. La coagulopatía asociada a COVID-19 es consecuencia de la tormenta de citocinas desencadenada por la respuesta inmune ante el SARS-CoV-2, la cual provoca lesión endotelial e hiperactivación de la cascada de la coagulación que culmina con generación de fibrina a nivel vascular, favoreciendo así, la estasis sanguínea. En la evaluación del paciente con infección por SARS-CoV-2, se considera al dímero $\mathrm{D}$ como el biomarcador de elección para el diagnóstico y monitoreo del estado de hipercoagulabilidad y el cual en el rango de $230 \mathrm{ng} / \mathrm{ml}-2000 \mathrm{ng} / \mathrm{ml}$, se ha asociado a mayor riesgo de enfermedad crítica $(66 \%)$, eventos trombóticos (38\%) y mortalidad $(47 \%)$. Otros métodos diagnósticos, aunque menos sensibles y específicos, son el nivel de fibrinógeno, tiempo parcial de tromboplastina y recuento plaquetario. Las principales complicaciones tromboembólicas en el paciente con SARS-CoV-2 son la tromboembolia pulmonar, trombosis venosa profunda, síndrome coronario agudo, evento cerebrovascular isquémico agudo, coagulopatía intravascular diseminada, isquemia mesentérica, trombosis aórtica abdominal y torácica; siendo el $80 \%$ tromboembolia pulmonar y $31 \%$ trombosis venosa profunda las más frecuentes.

\section{Referencias}

Alvarado Amador, I., Bandera Anzaldo, J., Carreto Binagui, L., Pavón Romero, G., \& Alejandre García, A. (2020). Etiología y fisiopatología del SARS-CoV-2. (3, Ed.) Revista Latinoamericana de Infectología Pediátrica , 33(1), s5-59. https:// doi.org/10.35366/96667

Avila, J., Long, B., Holladay, D., \& Gottlieb, M. (2021). Thromboembolic complications of COVID-19. The American Journal of Emergency Medicine, 39(Sólo resumen), 213-218. doi:https://doi.org/10.1016/j.ajem.2020.09.065

Barco-Moreno, A., Cuellar-Canizales, A., Vilas-Cruz, M., \& Bravo-Rojas, M. (2020). Revisión de tema: hipercoagulabilidad asociada a COVID-19, acercamiento clínico. Revista Salutem Scientia Spiritus, 6(1), 101-106. Recuperado el 30 de Junio de 2021, de https://revistas.javerianacali.edu.co/index.php/salutemscientiaspiritus/ article/download/2349/2898/ 
Cochrane,I.(2021). El dímeroDaltoseasociaconmayorgravedad deCOVID-19). Recuperado el 01 de Julio de 2021, de Cochrane lberoamérica: https://es.cochrane.org/ es/news/el-d\%C3\%ADmero-d-alto-se-asocia-con-mayor-gravedad-de-covid-19

Díaz-Castrillón, F., \& Toro-Montoya, A. (2020). SARS-CoV-2/COVID-19: el virus, la enfermedad y la pandemia. Revista Medicina \& Laboratorio, 24(3), 183-250. https://doi.org/10.36384/01232576.268

Espinosa-Rosales, F. (2020). Inmunopatología de la infección por virus SARS-CoV-2. Acta Pediátrica de México, 41(1), S42-s50. https://doi. org/10.18233/APM41No4S1ppS42-S502070

Gonzáles-Villalva, A., de la Peña-Díaz, A., Rojas-Lemus, M., López-Váldez, N., Ustarroz-Cano, M., García-Peldez, I., Fortoul, T. (2020). Fisiología de la hemostasia y su alteración por la coagulopatía en COVID-19. Revista de la Facultad de Medicina de la UNAM, 63(5), 45-57. https://doi.org/10.22201/ fm.24484865e.2020.63.5.08

Liao, S., Shao, S., Chen, Y., \& Hung, M. (2020). Incidence and mortality of pulmonary embolism in COVID-19: a systematic review and meta-analysis . Revista Critical Care, 24(464). https://doi.org/10.1186/s13054-020-03175-z

Mondal, S., Quintili, A., Karamchandani, K., \& Bose, S. (2020). Thromboembolic disease in COVID-19 patients: a brief narrative review. Journal of Intensive Care, 8, Artículo 70. https://doi.org/10.1186/s40560-020-00483-y

Rosa, C. (2020). Grupo Cooperativo Argentino de Hemostasia y Trombosis. Recuperado el 01 de Julio de 2021, de Grupo Cooperativo Argentino de Hemostasia y Trombosis: https://www.grupocaht.com/wp-content/uploads/2020/10/DD-y-COVID-19-Set-2020.pdf

Sáenz Morales, O., Rubio, A., Yomayusa, N., Gamba, N., \& Garay Fernández, M. (2020). Coagulopatía en la infección por el virus SARS-CoV-2 (COVID-19): de los mecanismos fisiopatológicos al diagnóstico y tratamiento. Acta Colombiana de Cuidado Intensivo, 285, 1-11. https://doi.org/10.1016/j.acci.2020.10.007 
Triana-Bedoya, G., \& León-Cardona, L. (2020). Tormenta de citoquinas y SARS-CoV-2. Revista Salutem Scientia Spiritus, 6(1), 161-166. Recuperado el 30 de Junio de 2021, de https://revistas.javerianacali.edu.co/index.php/salutemscientiaspiritus/ article/download/2345/2896/

Vargas-Lara, A., Schreiber-Vellnagel, V., Ochoa-Hein, E., \& López-Ávila, A. (2020). SARS-CoV-2: una revisión bibliográfica de los temas más relevantes y evolución del conocimiento médico sobre la enfermedad. Revista Neumología y Cirugía de Tórax, 79(3), 185-196. doi:https://dx.doi.org/10.35366/96655

Windyga,J.(2020). Guías: COVID-19ytrastornos delahemostasia: parte1. RevistaMedycyna Praktyczna, 7(8), 59-58. Recuperado el 01 de Julio de 2021, de https://empendium.com/ manualmibe/covid19/2442998,guias-covid-19-y-trastornos-de-la-hemostasia-parte-1

\section{Sobre la autora}

\section{Nurian Alejandra Hernández Loyo}

Es estudiante de la carrera de Médico y Cirujano del Centro Universitario de Oriente de la Universidad San Carlos de Guatemala y coautora de la investigación "Caracterización de pacientes VIH positivo con fallo virológico atendidos en Clínica de Atención Integral del Hospital Regional de Zacapa" en el año 2018 y autora de caso clínico interesante "Síndrome Nefrítico por Glomerulonefritis Aguda Postinfecciosa", aceptado para presentación en XXXVIII Congreso Nacional de Medicina Interna en el año 2020, organizado por la Asociación de Medicina Interna de Guatemala. 
Copyright (c) Nurian Alejandra Hernández Loyo

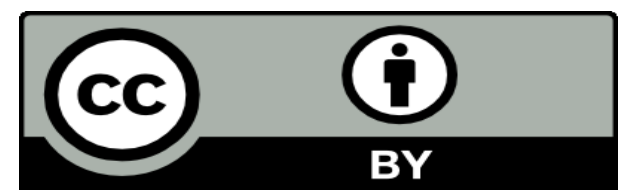

Este texto está protegido por una licencia CreativeCommons 4.0.

Usted es libre para compartir, copiar y redistribuir el material en cualquier medio o formato y adaptar el documento, remezclar, transformar y crear a partir del material para cualquier propósito, incluso comercialmente, siempre que cumpla la condición de atribución: usted debe reconocer el crédito de una obra de manera adecuada, proporcionar un enlace a la licencia, e indicar si se han realizado cambios. Puede hacerlo en cualquier forma razonable, pero no de forma tal que sugiera que tiene el apoyo del licenciante o lo recibe por el uso que hace. 